\title{
Carrier relaxation dynamics in InAs/InGaAlAs quantum dashes
}

\author{
A.I. Ryasnyanskiy ${ }^{\text {a,* }}$, I. Biaggio ${ }^{\text {a }}$, C.L. Tan ${ }^{\text {a }}$, H.S. Djie ${ }^{\text {a }}$, B.S. Ooi ${ }^{\text {a,b }}$ \\ a Lehigh University, Department of Physics, Bethlehem, PA 18015, USA \\ ${ }^{\mathrm{b}}$ King Abdullah University of Science \&' Technology (KAUST), Thuwal, Saudi Arabia
}

\section{A R T I C L E I N F O}

\section{Available online 29 October 2010}

\section{Keywords:}

Quantum dash

Carrier dynamics

Relaxation time

Four wave mixing

\section{A B S T R A C T}

We characterize size-dependent carrier relaxation dynamics of partial laser structures containing quantum dashes by time-resolved degenerate four wave mixing between 1.2 and $1.6 \mu \mathrm{m}$.

(c) 2010 Elsevier B.V. All rights reserved.

\section{Introduction}

Low-dimension semiconductor nanostructures have recently attracted much attention because of their wide physical interest and their potential device applications such as low current density threshold lasers [1,2], all-optical buffers [3,4], saturable absorbers [5], optical memories [6], etc. Among the variety of such materials the quantum dashes (strongly elongated quantum dots) are of growing interest now due to perspectives in optoelectronic applications, especially in telecommunication lasers operating at $1.55 \mu \mathrm{m}$ and longer wavelengths [7-9]. Size distribution of quantum dash (Q-dash) structures results in a broad gain profile and wide tunable emission (from 1.5 to $2 \mu \mathrm{m}$ ) [7].

One of the most important aspects of such nanostructures is the carrier dynamics that determines the criteria for effective operation of the optoelectronic devices.

Here we present measurements of the size-dependent carrier relaxation dynamics (CRD) of the partial laser structures containing quantum dashes (Q-dash), which have been obtained by studying transient grating dynamics in a time-resolved degenerate four wave mixing configuration.

\section{Experimental part}

\subsection{Optical properties of Q-dash materials}

The Q-dash partial laser structure was grown using a gas source molecular beam epitaxy on ( 100 ) oriented $n$-doped InP substrates. The active region consists of four sheets of 5 monolayer InAs dashes, each embedded within a $7.6 \mathrm{~nm}$ thick compressively strained $\operatorname{In}_{0.64} \mathrm{Ga}_{0.16} \mathrm{Al}_{0.2}$ As quantum well [8] with a thickness of

\footnotetext{
* Corresponding author. Tel.: +1 610759 3953; fax: +1 6107585730.

E-mail address: alr309@lehigh.edu (A.I. Ryasnyanskiy).
}

$50 \mathrm{~nm}$ of tensile strained $\operatorname{In}_{0.50} \mathrm{Ga}_{0.32} \mathrm{Al}_{0.18} \mathrm{As}$ barrier. The Q-dash structures have an average height of $3.2 \mathrm{~nm}$, average width of $18 \mathrm{~nm}$, and length varied from 20 to hundreds of nm. Photoluminescence (PL) spectroscopy was then performed at $77 \mathrm{~K}$ using a $980 \mathrm{~nm}$ diode laser as an excitation source on the $\mathrm{SiO}_{2}$ capped samples. The PL measurements at $1000 \mathrm{~W} / \mathrm{cm}^{2}$ of excitation source show (Fig. 1) almost equally-spaced ( $\sim 25 \mathrm{~nm}$ ) multiple emission humps, which indicates the presence of different quantized energy levels in the Q-dash system after a deconvolution study, as illustrated in Fig. 1. The Q-dash structure shows three major emission humps at $\sim 1520,1495$ and $1470 \mathrm{~nm}$, where each emission group possesses similar inhomogeneous broadening of $\sim 20 \mathrm{meV}$. The multiple almost equally spaced energy states could be attributed to the ground state emission from different Q-dash families across the stacking layers of active region.

\subsection{Carrier relaxation dynamics}

The CRD measurements have been done with pump and probe Degenerate-four wave mixing (DFWM) using 1 ps pulses in the wavelength range between 1200 and $1650 \mathrm{~nm}$ with a spectral width of about $2 \mathrm{~nm}$. The pulses are generated by a Light Conversion TOPAS parametric generator pumped by a Clark-MXR ${ }^{\mathrm{TM}}$ pulsed laser at a wavelength of $773 \mathrm{~nm}$. In our time-resolved DFWM experiment, the probe beam detects the presence of a transient grating induced by two pump beams, which delivers a clear and reliable signal. However, we note that the same results can be obtained in a conventional pump and probe measurement, proving that the signal that we detect originates from pump-pulse induced change in the optical absorption.

As discussed above, our samples have a wide size distribution of Q-dashes and can be considered as a complex of many Q-dash families of a particular size. One way to determine the influence of each family is to use the size-dependent absorption properties of Q-dashes. In a quantum confinement regime, large Q-dashes yield 


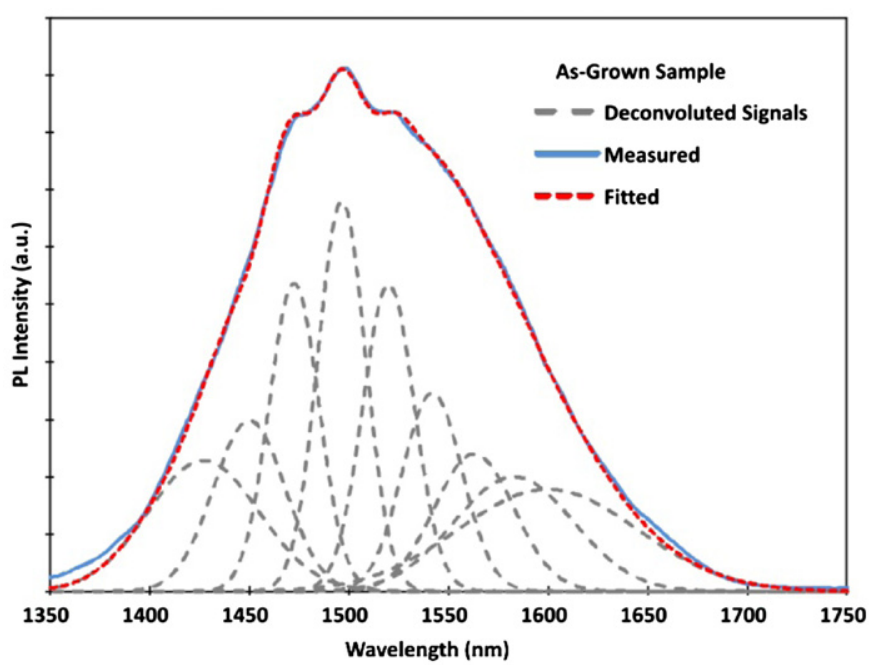

Fig. 1. PL emission from the as-grown Q-dash sample with the corresponding deconvoluted signals.

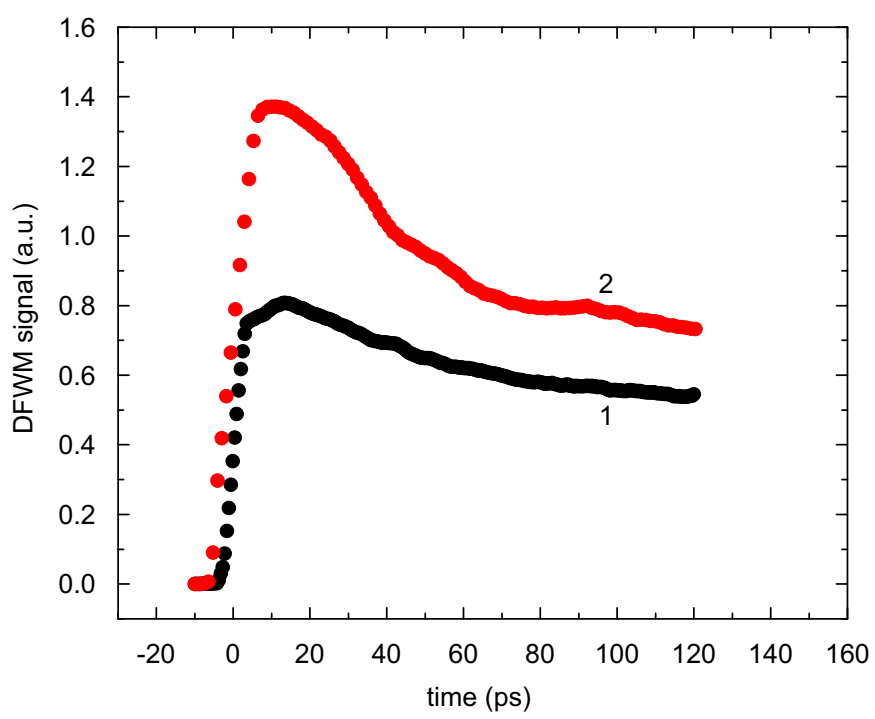

Fig. 2. Excited state population as determined by DFWM signal as a function of time delay for Q-dash sample on silica substrate (1) and Q-well structures at the wavelength of $1500 \mathrm{~nm}$ (2).

long-wavelength absorption, whereas the absorption wavelength will shift toward shorter wavelengths for small Q-dashes. Thus, tuning the excitation wavelength allows to study the CRD of a particular Q-dash family.

We performed two sets of DFWM experiments (i.e., when the sample was fixed to the silica substrate with refractive index matching oil between the two interfaces and in a free standing configuration). The reason for conducting of two sets of experiment will be described below.

\subsubsection{Q-dash sample on silica substrate.}

The results of the DFWM pump and probe measurement at the wavelength of $1500 \mathrm{~nm}$ are shown in the Fig. 2 for samples with Q-dashes and without Q-dashes (only quantum wells). One can see from the figure that the DFWM curves contain both fast and slow components. The experimental set-up did not allow us to measure the exact value of the slow component, which is of the order of several nanoseconds. At $1500 \mathrm{~nm}$, the fast components for pure

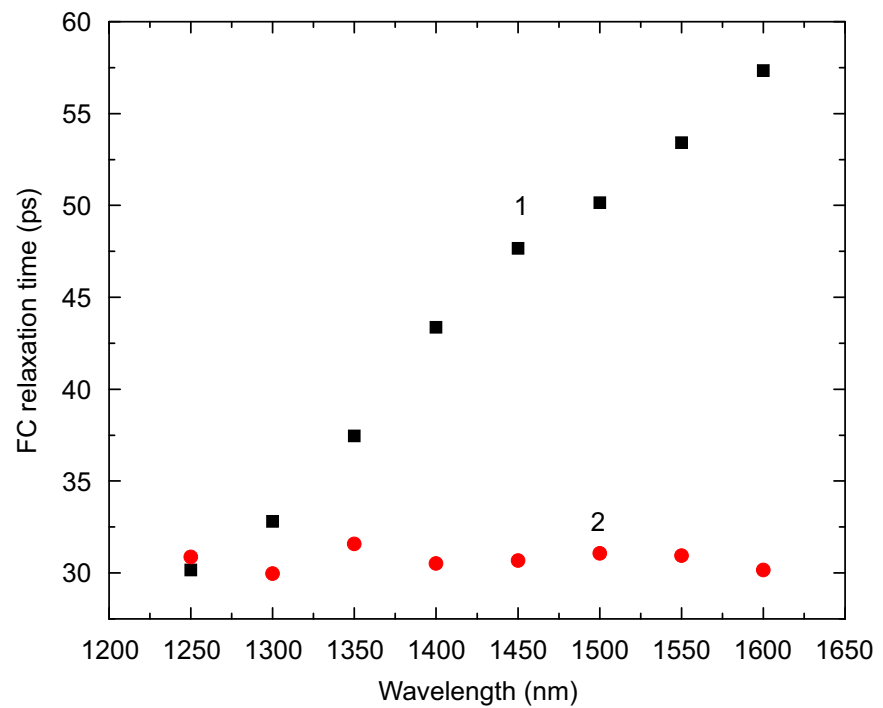

Fig. 3. Wavelength dependence of the fast relaxation component for Q-dash (1) and Q-well samples (2)

quantum well and Q-dash samples have an exponential relaxation time of 30 and $50 \mathrm{ps}$, respectively. Their dependence from the wavelength is presented in the Fig. 3. In Q-dashes, we observe an increase in the relaxation time from 30 up to $57 \mathrm{ps}$, while increasing the excitation wavelength from 1.25 to $1.6 \mu \mathrm{m}$. This change in the relaxation time is attributed to the influence of different Q-dash families. Longer wavelengths excite larger Q-dashes, leading to longer relaxation times.

We note that in sample without quantum dashes (pure quantum well structure), the free-carrier relaxation time was constant at $\sim 30 \mathrm{ps}$ in all investigated wavelength range (Fig. 3). Intensity dependent measurements $\left(5-15 \mathrm{MJ} \mathrm{cm}^{-2}\right)$ did not show any significant difference in carrier dynamics in both Q-dashes and quantum-well only samples.

We attribute the fast relaxation component to free carriers and the slow component to trapped carriers, which is a common situation in semiconductor nanostructures and was previously observed in quantum dot systems $[10,11]$. Size dependence of the fast decay component can be understood by considering surface states that act as recombination centers. Increase of the size of Q-dashes leads to decrease of the surface/volume ratio and therefore the fast decay component becomes slower.

The slow component (1-2 ns) has the order of magnitude of the exciton radiation lifetime measured in similar structures $[12,13]$ and therefore can be connected with electron-hole recombination in Q-dashes.

\subsubsection{Free standing $Q$-dash sample.}

In the free standing Q-dash sample we observed an additional oscillatory time dynamics accompanying the decay in the whole investigated wavelength window (1250-1650 nm). The results for $1500 \mathrm{~nm}$ are shown in Fig. 4 as an example. The oscillation period was $\sim 45$ ps for all the measurements. No change in oscillatory behavior was observed with intensity of the excitation. By varying our experimental configurations, we found that these oscillations can only be obtained in free-standing samples, where the excitation and probe beams enter the sample from the side where the Q-dashes are. Flipping the sample around, as well as creating an optical contact of the back surface with a glass surface, reliably removes the oscillations. From this we conclude that these oscillations are related to the reflection of the input beams from 


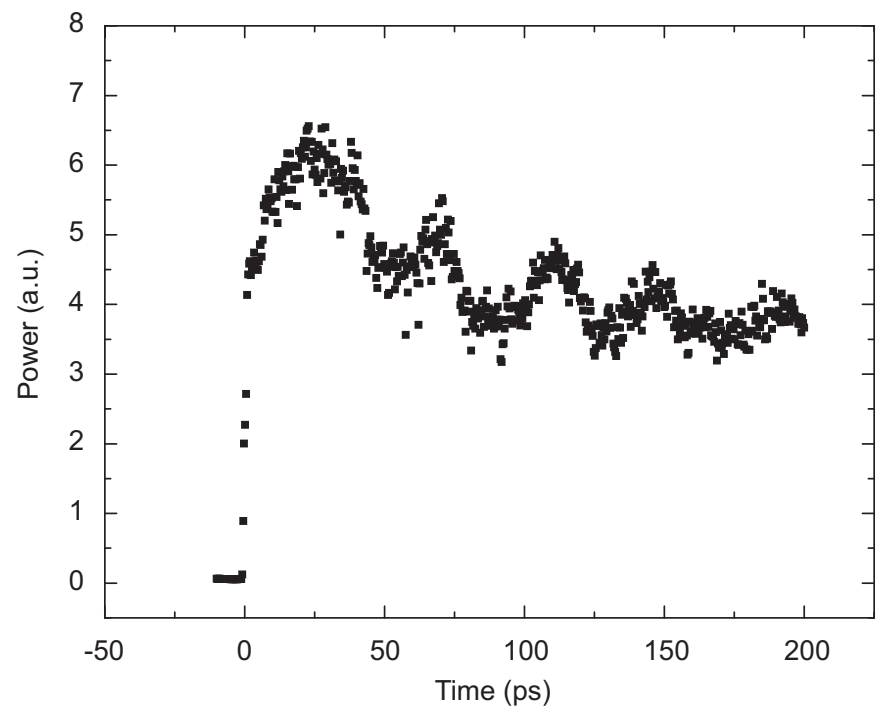

Fig. 4. Excited state population as determined by DFWM signal as a function of time delay for free standing Q-dash sample at the wavelength of $1500 \mathrm{~nm}$.

the back surface of the sample (which with refractive index of 3.5 has a reflectivity of $30 \%$ ). However, their time-period is not related to time-of-flight effects because the period is the same for various sample thicknesses. Neither can they be explained with quantum interference or mechanical oscillations: oscillatory signals originating from quantum beats or laser induced ultrasound would have periods much shorter or much longer, respectively, than those we observed. The evaluation of the origin of this oscillatory signal is still a work in progress and it will be the subject of a future publication.

\section{Conclusion}

We identified size-dependent carrier relaxation times in Q-dashes grown by molecular beam epitaxy on $\left(\begin{array}{lll}1 & 0 & 0\end{array}\right)$ oriented n-doped InP substrates. These relaxation times are caused by fast surface recombination and vary from $30-57$ ps for Q-dash sizes in the range of $2-4 \mathrm{~nm}$ and slow electron-hole recombination (1-2 ns). In addition we observed that the absorption of the quantum-dash samples oscillates with time with a period of the order of 45 ps when excitation and probing of the sample is done with pulses entering the Q-dash surface first.

\section{References}

[1] P. Caroff, C. Paranthoen, C. Platz, O. Dehaese, H. Folliot, N. Bertru, C. Labbé, R. Piron E. Homeyer, A. Le Corre, S. Loualiche, Appl. Phys. Lett. 87 (2005) 243107.

[2] Jin-Hong-Lee Jin-Soo-Kim, Won-Seok-Han Sung-Ui-Hong, Ho-Sang-Kwack, Chul-Wook-Lee, Dae-Kon-Oh, IEEE Phot. Technol. Lett. 16 (2004) 1607.

[3] C.J. Chang-Hasnain, P.-Ch. Ku, J. Kim, S.-L. Chuang, Proc. IEEE. 91 (2003) 1884

[4] R.S. Tucker, P.-Ch. Ku, C.J. Chang-Hasnain, J. Light. Technol. 23 (2005) 4046.

[5] J.G. Cederberg, D.A. Bender, M.V. Pack, R.L. Schmitt, J. Cryst. Growth 312 (2010) 1375.

[6] K. Imamura, Y. Sugiyama, Y. Nakata, Sh. Muto, N. Yokoyama, Jpn. J. Appl. Phys. 34 (1995) L1445.

[7] W.R-. Rudzinski, R. Kudrawiec, P. Podemski, G. Sek, J. Misiewicz, A. Somers R. Schwertberger, J.P. Reithmaier, A. Forchel, Appl. Phys. Lett. 89(2006) 031908

[8] P. Podemski, G. Sek, K. Ryczko, J. Misiewicz, S. Hein, S. Hofling, A. Forchel G. Patriarche, Appl. Phys. Lett. 93 (2008) 171910.

[9] H.S. Djie, C.L. Tan, B.S. Ooi, J.C.M. Hwang, X. -M. Fang, Y. Wu, J.M. Fastenau, W.K. Liu, G.T. Dang, W.H. Chang, Appl. Phys. Lett. 91 (2007) 111116.

[10] T. Okuno, Y. Masumoto, M. Ikezawa, T. Ogawa, A. Lipovskii, Appl. Phys. Lett. 77 (2000) 504.

[11] I. Yagi, K. Mikami, K. Ebina, M. Okamura, K. Uosaki, J. Phys. Chem. B 110 (2006) 14192.

[12] A. Melliti, M.A. Maaref, F. Hassen, M. Hjiri, H. Maaref, J. Tignon, B. Sermage, Solid State Commun 128 (2003) 213.

[13] C. Cornet, C. Labbé, H. Folliot, P. Caroff, C. Levallois, O. Dehaese, J. Even, A. Le Corre, S. Loualiche, Appl. Phys. Lett. 88 (2006) 171502. 